\title{
A New CMM Based Method of Camera Calibration and Distortion Compensation
}

\author{
Shugui Liu, Zhenhua Han, Sen Wang, Guoxiong Zhang \\ State Key Laboratory of Precision Measuring Technology and Instruments, Tianjin University, Tianjin, \\ 300072, China, \\ hanzhenhua@tju.edu.cn
}

Keywords: high-precision, camera calibration, distortion compensation, CMM, distortion database, flexibility.

\begin{abstract}
To improve the accuracy of vision measuring system, high-precision camera calibration and distortion compensation are essential. A new CMM based method of camera calibration and distortion compensation, and a new index for evaluating the residual distortion after compensation are presented in this paper. A three-dimensional reference with a large number of feature points is provided by a high accurate CMM. Such a $3 \mathrm{D}$ reference is flexible in terms of the spacing and distribution of feature points and is able to cover the entire image plane of the camera. The internal and external parameters of the camera are obtained through nonlinear optimization. A distortion database is established based on the calibrated data to compensate the residual errors. Experiments show that the new method has higher accuracy and flexibility in comparison with commonly used methods, and can be applied for calibrating cameras in various vision measuring systems.
\end{abstract}

\section{Introduction}

Camera calibration is essential in vision measuring systems. The accuracy of camera calibration and distortion compensation directly affects the accuracy of vision measuring system. The research on camera lens distortion can be traced back to 1919, when A. Conrady first introduced the decentering distortion [4], and afterwards many effective methods have been proposed. These methods can be roughly classified into three categories: linear methods, nonlinear methods and combined methods.

Linear methods involve the determination of transformation parameters by solving linear equations, whose main advantages are simple and fast in data processing. However lens distortion is not considered and the solution is noise sensitive [11]. Direct linear transformation was introduced by Abdal-Aziz and Karara in 1970's, nevertheless its accuracy is not high enough.

Nonlinear methods establish a nonlinear mathematical model with a large number of unknowns. These methods may achieve high accuracy through nonlinear optimization. However the algorithm is complicated and a bad initial guess may lead to unstable results. References [8], [9], [10], [15], [16] belong to such methods. Self-calibration methods are based on the corresponding relationship among a large number of pictures taken $[12,13]$. They are flexible and can be used in a variety of cases. But they are not so robust.

Methods which combine linear and nonlinear transformations are widely used currently. The RAC method introduced by Tsai [7] is a typical approach. Its algorithm is relatively simple, and each parameter can be solved through an iterative optimization. However only the radial distortion is taken into account and it leads to unsatisfactory results in distortion compensation in many cases. A flexible camera calibration method [14] was proposed by Zhang, in which the constraints are established based on checkerboard images with different attitudes. It uses linear equations to solve distortion coefficients and nonlinear optimization afterwards. Later on, different mathematical models for calibrating radial distortion, centrifugal distortion, thin prism distortion and others are put forward by researchers [1], [2], [3], [5], [11], [14]. These techniques improved the accuracy of distortion compensation to a certain extent. However the large scale vision measuring systems ask for even 
higher accuracy of distortion compensation. Most common used calibration methods use a plane target as the calibration reference and the images of feature points generally can cover only part of the image plane.

In order to further improve the accuracy of camera calibration and distortion compensation, a new coordinate measuring machine $(\mathrm{CMM})$ based method of high-precision camera calibration and distortion compensation is presented in this paper. A large number of three-dimensional reference feature points can be established and their images are able to cover the entire image plane of the camera. A distortion database is established based on the calibrated data to compensate the residual errors and a new index to evaluate the results of distortion compensation is proposed.

\section{Imaging Distortion Model of the Camera}

Errors of the CCD readings, image errors, and calibration errors, errors caused by the distortion of optical system and environment conditions are the main error sources of a camera system. Only the calibration errors and distortions of the optical system will be discussed in this paper. Both these two types of errors can be considered as calibration errors, since errors caused by distortions of the optical system can be compensated by accurate calibration in a large degree. The camera distortion includes radial distortion, centrifugal distortion, thin prism distortion and some others. The distortions in $x$ and $y$ directions can be expressed as [18]

$$
\begin{aligned}
& \delta_{x}=x_{\mathrm{r}} k_{1}\left(x_{\mathrm{r}}^{2}+y_{\mathrm{r}}^{2}\right)+x_{\mathrm{r}} k_{2}\left(x_{\mathrm{r}}^{2}+y_{\mathrm{r}}^{2}\right)^{2}+\cdots+p_{1}\left(3 x_{\mathrm{r}}^{2}+y_{\mathrm{r}}^{2}\right)+2 p_{2} x_{\mathrm{r}} y_{\mathrm{r}}+s_{1}\left(x_{\mathrm{r}}^{2}+y_{\mathrm{r}}^{2}\right) \\
& \delta_{y}=y_{\mathrm{r}} k_{1}\left(x_{\mathrm{r}}^{2}+y_{\mathrm{r}}^{2}\right)+y_{\mathrm{r}} k_{2}\left(x_{\mathrm{r}}^{2}+y_{\mathrm{r}}^{2}\right)^{2}+\cdots+p_{2}\left(x_{\mathrm{r}}^{2}+3 y_{\mathrm{r}}^{2}\right)+2 p_{1} x_{\mathrm{r}} y_{\mathrm{r}}+s_{2}\left(x_{\mathrm{r}}^{2}+y_{\mathrm{r}}^{2}\right)
\end{aligned}
$$

In Eq. (1) $k_{1}, k_{2}$ are coefficients of radial distortion, $p_{1}, p_{2}$ are coefficients of centrifugal distortion and $s_{1}, s_{2}$ are coefficients of thin prism distortion, $\delta_{x}$ and $\delta_{y}$ are distortions in $x$ and $y$ directions respectively, $\left(x_{\mathrm{r}}, y_{\mathrm{r}}\right)$ are nominal coordinates of image points. Centrifugal distortion and thin prism distortion can be generally classified as the tangential distortion [4]. Besides, the focal length $f$, coordinates $\left(c_{x}, c_{y}\right)$ of the principal point, external camera parameters and the pixel size of image in $x$ direction should be also calibrated. Form errors of the lens, squareness error of the image plane with reference to the optical axis and offsets of the array center with reference to the optical principal point, i. e. the intersection point of the optical axis with the image plane, are main sources causing camera distortion [5]. Some errors are irregular to a certain extent.

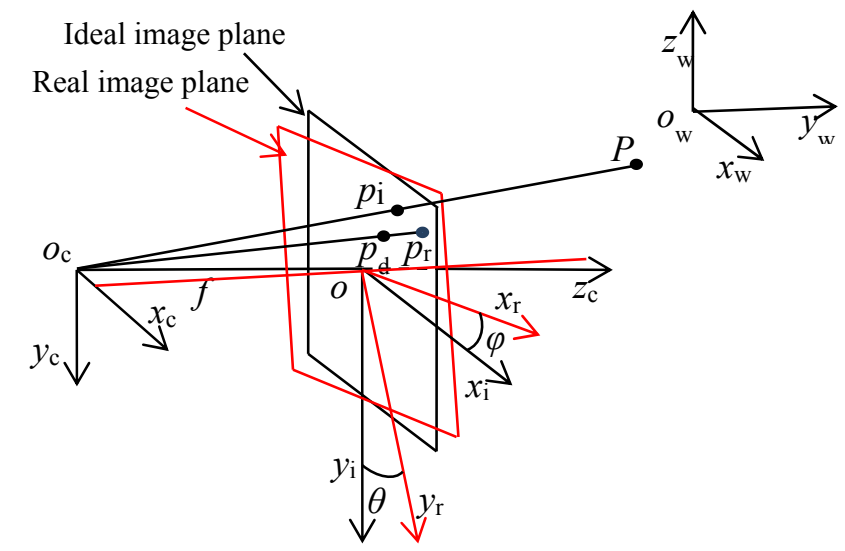

Fig. 1 The real and ideal imaging models

Based on the ideal pinhole imaging model an ideal coordinate system is established, as shown by solid black lines in Fig. 1. The real image plane and optical axis are described by red lines. There are certain rotational angles between the real image plane and ideal one. The real image plane coordinate system is converted to the ideal image plane coordinate system through a rotational transformation with angles $\theta$ and $\varphi$. Tangential distortion can be represented by this rotation transformation [4]. The values of $\theta, \varphi, k_{1}, k_{2}$ can be determined based on that all the calibrated points on a straight line remain collinear in the ideal imaging model. By knowing rotation angles $\theta, \varphi$ and radial distortion coefficients $k_{1}, k_{2}$ coordinates of ideal image points are derived from calibrated data. Most of the camera calibration and distortion compensation methods are based on this model. 
Let $o_{\mathrm{w}}-x_{\mathrm{w}} y_{\mathrm{w}} z_{\mathrm{w}}$ represent the world coordinate system, $o_{\mathrm{c}}-x_{\mathrm{c}} y_{\mathrm{c}} z_{\mathrm{c}}$ the camera coordinate system, $o-x_{\mathrm{i}} y_{\mathrm{i}}$ the ideal image plane coordinate system, and $o-x_{\mathrm{r}} y_{\mathrm{r}}$ the real image plane coordinate system. $P$ is a point in the real space, $p_{\mathrm{i}}$ is its ideal image, $p_{\mathrm{r}}$ is its real image, and $p_{\mathrm{d}}$ is its image with radial distortion. The $3 \mathrm{D}$ coordinate system $o-z_{\mathrm{c}} x_{\mathrm{i}} y_{\mathrm{i}}$ is established based on the ideal image plane coordinate system $o-x_{\mathrm{i}} y_{\mathrm{i}}$ and the optical axis $z_{\mathrm{c}}$ of the camera,

\section{A New Method of Camera Calibration and Distortion Compensation}

\subsection{Theory}

In Fig. $1\left(u_{\mathrm{r}}, v_{\mathrm{r}}\right)$ are coordinates of point $p_{\mathrm{r}}$ in the real image plane coordinate system $o-u_{\mathrm{r}} v_{\mathrm{r}}$ expressed in pixels. $\left(x_{\mathrm{r} 0}, y_{\mathrm{r} 0}\right)$ are those expressed in millimeters. Their conversion relationship can be formulated as.

$$
\left[\begin{array}{l}
x_{\mathrm{r} 0} \\
y_{\mathrm{r} 0}
\end{array}\right]=\left[\begin{array}{cc}
d_{x} & 0 \\
0 & d_{y}
\end{array}\right]\left[\begin{array}{l}
u_{\mathrm{r}}-c_{x} \\
v_{\mathrm{r}}-c_{y}
\end{array}\right]
$$

Where $\left(c_{x}, c_{y}\right)$ are coordinates of the principal point in the real image plane coordinate system expressed in pixels. $d_{x}$ and $d_{y}$ are the pixel sizes of the CCD in $x$ and $y$ directions, respectively. The coordinates of point $p_{\mathrm{r}}\left(x_{\mathrm{r} 0} 0, y_{\mathrm{r} 0}, 0\right)$ in system $o-x_{\mathrm{r}} y_{\mathrm{r}} z_{\mathrm{r}}$ are transformed to $\left(x_{\mathrm{r},} y_{\mathrm{r}}, z_{\mathrm{r}}\right)$ in system $o-x_{\mathrm{i}} y_{\mathrm{i}} z_{\mathrm{c}}$ by.

$$
\left[\begin{array}{c}
x_{\mathrm{r}} \\
y_{\mathrm{r}} \\
z_{\mathrm{r}}
\end{array}\right]=\boldsymbol{R}_{\mathrm{c}}\left[\begin{array}{c}
x_{\mathrm{r} 0} \\
y_{\mathrm{r} 0} \\
0
\end{array}\right]
$$

Where $z_{\mathrm{r}}$ is perpendicular to plane $o-x_{\mathrm{r}} y_{\mathrm{r}}$ and the system $o-x_{\mathrm{r}} y_{\mathrm{r}} z_{\mathrm{r}}$ obeys right hand rule.

Since rotation angles between the real and ideal image planes are small, the rotation matrix $\boldsymbol{R}_{\mathrm{c}}$ can be simplified as

$$
\boldsymbol{R}_{\mathrm{c}} \approx\left[\begin{array}{ccc}
1 & 0 & \varphi \\
0 & 1 & -\theta \\
-\varphi & \theta & 1
\end{array}\right]
$$

Coordinates $p_{\mathrm{d}}\left(x_{\mathrm{d}} y_{\mathrm{d}}\right)$ in the camera coordinate system $o_{\mathrm{c}}-x_{\mathrm{c}} y_{\mathrm{c}} z_{\mathrm{c}}$ can be expressed as

$$
x_{\mathrm{d}}=\frac{f}{f+z_{\mathrm{r}}} x_{\mathrm{r}} \quad y_{\mathrm{d}}=\frac{f}{f+z_{\mathrm{r}}} y_{\mathrm{r}}
$$

Coordinates $\left(x_{\mathrm{i}}, y_{\mathrm{i}}\right)$ in the ideal image plane coordinate system $o u_{\mathrm{i}} v_{\mathrm{i}}$ can be written as

$$
\begin{aligned}
& x_{\mathrm{i}}=x_{\mathrm{d}}+\delta_{k x} \quad y_{\mathrm{i}}=y_{\mathrm{d}}+\delta_{k y} \\
& \delta_{k x}=x_{\mathrm{d}} k_{1}\left(x_{\mathrm{d}}^{2}+y_{\mathrm{d}}^{2}\right)+x_{\mathrm{d}} k_{2}\left(x_{\mathrm{d}}^{2}+y_{\mathrm{d}}^{2}\right)^{2} \\
& \delta_{k y}=y_{\mathrm{d}} k_{1}\left(x_{\mathrm{d}}^{2}+y_{\mathrm{d}}^{2}\right)+y_{\mathrm{d}} k_{2}\left(x_{\mathrm{d}}^{2}+y_{\mathrm{d}}^{2}\right)^{2}
\end{aligned}
$$

$\delta_{k x}$ and $\delta_{k y}$ are radial distortion compensation values in $x_{\mathrm{i}}, y_{\mathrm{i}}$ directions, respectively. Based on the pinhole camera model, coordinates of point $p\left(x_{\mathrm{c}}, y_{\mathrm{c}}, z_{\mathrm{c}}\right)$ in the camera coordinate system $o_{\mathrm{c}}-x_{\mathrm{c}} y_{\mathrm{c}} z_{\mathrm{c}}$ can be expressed as

$$
x_{\mathrm{i}}=\frac{f}{z_{\mathrm{c}}} x_{\mathrm{c}} \quad y_{\mathrm{i}}=\frac{f}{z_{\mathrm{c}}} y_{\mathrm{c}}
$$

The coordinates of point $P$ in world system $o_{\mathrm{w}}-x_{\mathrm{w}} y_{\mathrm{w}} z_{\mathrm{w}}$ can be converted to those in camera system $o_{\mathrm{c}}-x_{\mathrm{c}} y_{\mathrm{c}} z_{\mathrm{c}}$ by

$$
\left(\begin{array}{l}
x_{\mathrm{c}} \\
y_{\mathrm{c}} \\
z_{\mathrm{c}}
\end{array}\right)=\boldsymbol{R}\left(\begin{array}{c}
x_{\mathrm{w}} \\
y_{\mathrm{w}} \\
z_{\mathrm{w}}
\end{array}\right)+\boldsymbol{T}
$$

where the translation vector $\boldsymbol{T}=\left[t_{x} t_{y} t_{z}\right]^{\mathrm{T}}$ and rotational matrix

$$
\boldsymbol{R}=\left(\begin{array}{lll}
r_{1} & r_{2} & r_{3} \\
r_{4} & r_{5} & r_{6} \\
r_{7} & r_{8} & r_{9}
\end{array}\right)=\left(\begin{array}{ccc}
\cos \beta \cos \gamma & \sin \alpha \sin \beta \cos \gamma-\cos \alpha \sin \gamma & \cos \alpha \sin \beta \cos \gamma+\sin \alpha \sin \gamma \\
\cos \beta \sin \gamma & \sin \alpha \sin \beta \sin \gamma+\cos \alpha \cos \gamma & \cos \alpha \sin \beta \sin \gamma-\sin \alpha \cos \gamma \\
-\sin \beta & \sin \alpha \cos \beta & \cos \alpha \cos \beta
\end{array}\right)
$$

in which $\alpha, \beta, \gamma$ are angles rotated about $x_{\mathrm{c}}, y_{\mathrm{c}}$ and $z \mathrm{c}$, respectively. 
To determine all the unknown internal and external parameters of the camera the following object function is built

$$
F_{0}\left(\alpha, \beta, \gamma, t_{x}, t_{y}, t_{z}, f, d_{x}, k_{1}, k_{2}, c_{x}, c_{y}\right)=\sum_{j=1}^{n} f_{a j}^{2}+\sum_{j=1}^{n} f_{b j}^{2}
$$

where

$$
\begin{aligned}
& f_{d j}=\left(r_{1} x_{\mathrm{w}}+r_{2} y_{\mathrm{w}}+r_{3} z_{\mathrm{w}}+t_{x}\right) f-\left(r_{7} x_{\mathrm{w}}+r_{8} y_{\mathrm{w}}+r_{9} z_{\mathrm{w}}+t_{z}\right)\left(\frac{f x_{\mathrm{r} 0}}{f+\theta y_{\mathrm{r} 0}-\varphi x_{\mathrm{r} 0}}+\delta_{k x}\right) \\
& f_{b j}=\left(r_{4} x_{\mathrm{w}}+r_{5} y_{\mathrm{w}}+r_{6} z_{\mathrm{w}}+t_{y}\right) f-\left(r_{7} x_{\mathrm{w}}+r_{8} y_{\mathrm{w}}+r_{9} z_{\mathrm{w}}+t_{z}\right)\left(\frac{f_{\mathrm{r} 0}}{f+\theta y_{\mathrm{r} 0}-\varphi x_{\mathrm{r} 0}}+\delta_{h y}\right)
\end{aligned}
$$

The first part of $f_{a j}$ is the product of $f$ and $x_{\mathrm{c}}$ determined by coordinates of calibrated point $P$ in space through $\boldsymbol{R}, \boldsymbol{T}$ transformations. The second part of $f_{a j}$ is the product of $z_{\mathrm{c}}$ and $x_{\mathrm{i}}=\left(x_{\mathrm{d}}+\delta_{k x}\right)$. In the ideal case they should be equal in accordance with Eq. (7). The difference between $x_{\mathrm{c}} f$ and $z_{\mathrm{c}} x_{\mathrm{i}}$ is taken as $f_{a j}$. The first part of $f_{b j}$ is the product of $f$ and $y_{\mathrm{c}}$ determined by coordinates of calibrated point $P$ in space through $\boldsymbol{R}, \boldsymbol{T}$ transformations. The second part of $f_{b j}$ is the product of $z_{\mathrm{c}}$ and $y_{\mathrm{i}}=\left(y_{\mathrm{d}}+\delta_{k y}\right)$. In the ideal case they should be equal. The sum of their squared differences serves as the objective function.

According to [6] $d_{y}$ is not needed to be optimized. Levenberg-Marquardt optimization algorithm is used here in two steps. In the first step parameters $\alpha, \beta, \gamma, t_{x}, t_{y}, t_{z}, f, d_{x}, k_{1}, k_{2}, c_{x}, c_{y}$ are optimized. Initial values of coefficients $k_{1}, k_{2}$ are zero. Initial coordinates $\left(c_{x}, c_{y}\right)$ of the principal point are those of the central pixel on the image plane. Initial value of $d_{x}$ is given by the camera manufacturer in its manual. The positional relationship between the camera coordinate system and the world system is shown in Fig. 1. Initial values of $\alpha, \beta, \gamma$ are $0.5 \pi, 0$ and 0 , respectively. Initial values of $t_{x}, t_{y}, t_{z}$ are estimated by some measuring scales. Both initial values of $\theta, \varphi$ are zero.

In the second step results of the first step are substituted in Eq. (11) as known values. Then the objective function $F_{1}$ is optimized for determining the rotation angles $\theta, \varphi$.

$$
F_{1}(\theta, \phi)=\sum_{j=1}^{n} f_{a j}^{2}+\sum_{j=1}^{n} f_{b j}^{2}
$$

Iteration is carried out for optimizing all the camera parameters until the value of objective function $F_{0}$ becomes less than a given threshold.

\subsection{Collecting Calibration Data}

A CMM based method for camera calibration is presented in this paper. The new method can provide a large number of three-dimensional control points flexibly with desired range and pitches. It is able to cover the entire image plane of the camera and provide more comprehensive three-dimensional feature information. The software applied to control the CMM and camera is POSCOM developed by ourselves. A circular light point is formed by a LED fixed on the ram of the CMM as shown in Fig. 2.

In order to obtain more three-dimensional spatial information, the data are sampled on three parallel planes as shown in Fig. 3. To minimize the impact of temperature variation, the calibration is carried out in a temperature controlled laboratory. To achieve high accuracy and reduce the errors caused by temperature drifts of the CMM and camera with time, $100 \times 100$ points are sampled on plane $b_{2}$, and $10 \times 10$ points are sampled on planes $b_{1}$ and $b_{3}$, respectively. The total time required for collecting 10200 data points is about 2 hours. The internal parameters of the camera keep effective when it is used in a shop floor. Its external parameters should be recalibrated in-situ. 


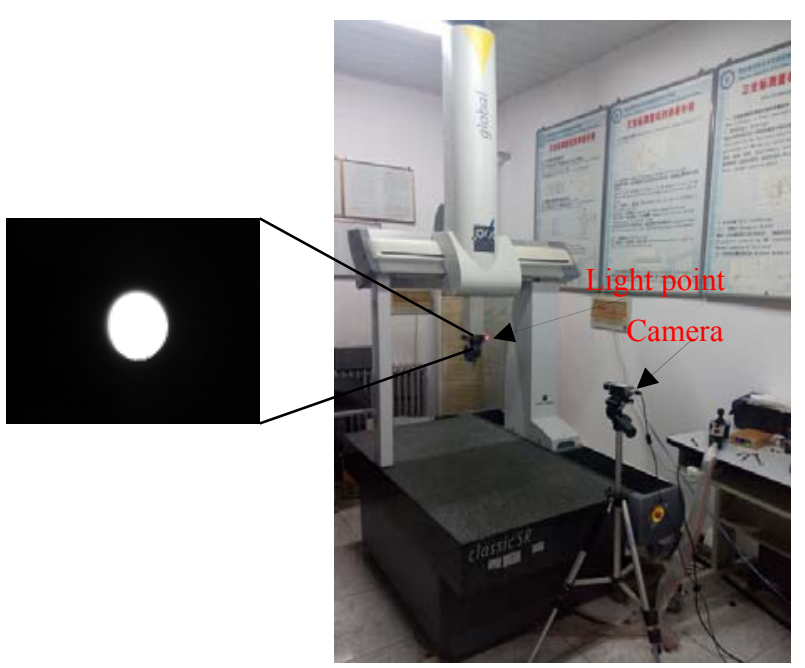

Fig. 2 The experimental setup

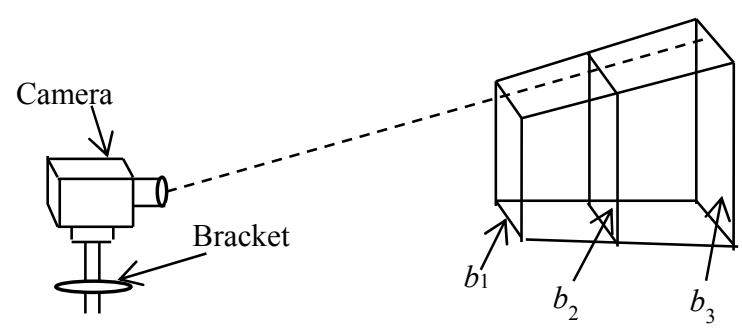

Fig. 3 The scheme of data collection space

\subsection{Distortion Database Compensation}

Due to the imperfection of the distortion model expressed by Eq. (1) some residual errors will remain after compensation based on this model. A distortion database for compensating the residual distortion is established. Residual distortions dif $x$, dify are differences between coordinates $\left(x_{\mathrm{i}}, y_{\mathrm{i}}\right)$ of the ideal image point and corresponding coordinates ( $\left.x_{\mathrm{if}}, y_{\mathrm{if}}\right)$ compensated by the distortion model. Coordinates $\left(x_{\mathrm{i}}, y_{\mathrm{i}}\right)$ are calculated from world coordinates $\left(x_{\mathrm{w}}, y_{\mathrm{w}}, z_{\mathrm{w}}\right)$ of the reference point provided by the CMM and rotation matrix $\boldsymbol{R}$, translation vector $\boldsymbol{T}$, as well as focal length $f$ in accordance with Eq. (13).

$$
\begin{gathered}
x_{\mathrm{i}}=\frac{\left(r_{1} x_{\mathrm{w}}+r_{2} y_{\mathrm{w}}+r_{3} z_{\mathrm{w}}+t_{x}\right) f}{r_{7} x_{\mathrm{w}}+r_{8} y_{\mathrm{w}}+r_{9} z_{\mathrm{w}}+t_{z}} \\
y_{\mathrm{i}}=\frac{\left(r_{4} x_{\mathrm{w}}+r_{5} y_{\mathrm{w}}+r_{6} z_{\mathrm{w}}+t_{y}\right) f}{r_{7} x_{\mathrm{w}}+r_{8} y_{\mathrm{w}}+r_{9} z_{\mathrm{w}}+t_{z}}
\end{gathered}
$$

Coordinates $\left(x_{\mathrm{if}}, y_{\mathrm{if}}\right)$ are calculated for all corresponding points on plane $b_{2}$ compensated by distortion model

$$
\begin{aligned}
& \operatorname{dif}_{x}=x_{\mathrm{i}}-x_{\mathrm{if}} \\
& \operatorname{dif}_{y}=y_{\mathrm{i}}-y_{\mathrm{if}}
\end{aligned}
$$

First, the sampled coordinates of particular image point are compensated by the distortion model. However in most cases the sampled point $p_{i}$ on the image plane is not exactly on the grid of the distortion database. Four nearest grid points $A, B, C, D$ around sampled point $p_{\text {i }}$ are found as shown in Fig. 4 and their corresponding residual distortions dif $f_{x}$, dify are obtained from the database. Linear interpolation is made for the sampled point $p_{\mathrm{i}}$. In Fig. 4 coordinate system $o-x_{\mathrm{i}} y_{\mathrm{i}}$ is the ideal image plane coordinate system. The length of the line perpendicular to the plane $o x_{i} y_{i}$ represents the residual distortion in $x$ direction of particular point. Lengths of line segments $A A_{1}, B B_{1}, C C_{1}, D D_{1}$ are residual distortions of corresponding grid points $A, B, C, D$. Lengths of line segments $E E_{1}, F F_{1}$ are those of corresponding interpolation points $E, F$. The length of line segment $p_{\mathrm{i}} p_{\mathrm{i} 1}$ is residual distortion difpx of 
point $p_{\mathrm{i}}$ in $x$ direction. All they can be calculated from Eq. (15). The residual distortion dif $f_{p y}$ of point $p_{\mathrm{i}}$ in $y$ direction can be determined in a similar way.

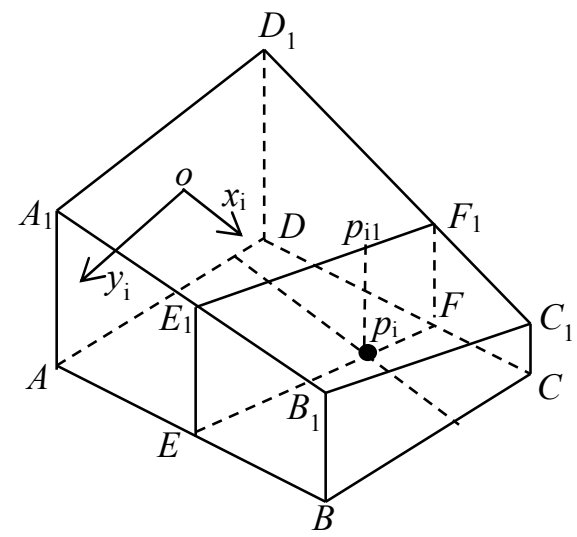

Fig. 4 Linear interpolation

$$
\begin{aligned}
& E E_{1}=B B_{1}\left(\frac{A E}{A B}\right)+A A_{1}\left(\frac{B E}{A B}\right) \\
& F F_{1}=C C_{1}\left(\frac{D F}{D C}\right)+D D_{1}\left(\frac{C F}{D C}\right) \\
& d i f_{p x}=E E_{1}\left(\frac{p_{\mathrm{i}} F}{E F}\right)+F F_{1}\left(\frac{E p_{\mathrm{i}}}{E F}\right)
\end{aligned}
$$

\section{Experiment}

\subsection{An Example of the Distortion Database}

The CCD with model Basler piA2400-17gm used in our experiment has a resolution of $2448 \times 2050$, and pixel size $3.45 \mu \mathrm{m} \times 3.45 \mu \mathrm{m}$. The focal length of the lens with model PENTAXC1614-M is $16 \mathrm{~mm}$. The distortion database is based on 10200 feature points with distribution shown in Fig. 3. The internal and external parameters of the camera obtained from calibration are shown in Table 1.

Table 1. Camera parameters

\begin{tabular}{|c|c|c|c|}
\hline$f[\mathrm{~mm}]$ & 16.014 & $\varphi[\mathrm{rad}]$ & $-5.6 \times 10^{-6}$ \\
\hline$c_{x}[\mathrm{pixel}]$ & 1246.18 & $\alpha[\mathrm{rad}]$ & 1.5708 \\
\hline$c[\mathrm{pixel}]$ & 1029.75 & $\beta[\mathrm{rad}]$ & $6.9 \times 10-4$ \\
\hline$d_{x}[\mathrm{~mm}]$ & $3.45 \times 10^{-3}$ & $\gamma[\mathrm{rad}]$ & $-1.79 \times 10^{-3}$ \\
\hline$k_{1}$ & $6.0 \times 10^{-4}$ & $t_{x}[\mathrm{~mm}]$ & -265.991 \\
\hline$k_{2}$ & $-5.0 \times 10^{-6}$ & $t_{y}[\mathrm{~mm}]$ & -339.831 \\
\hline$\theta[\mathrm{rad}]$ & $-2.9 \times 10^{-4}$ & $t_{z}[\mathrm{~mm}]$ & 514.139 \\
\hline
\end{tabular}

Distortion database consists of image plane coordinates $\left(x_{\mathrm{i}}, y_{\mathrm{i}}\right)$ of grid points and residual distortion compensation values dif $f_{x}$, dif $y$. Both image plane coordinates $\left(x_{\mathrm{i}}, y_{\mathrm{i}}\right)$ and $\left(x_{\mathrm{if}}, y_{\mathrm{if}}\right)$ of grid points are converted into pixel coordinates $\left(u_{\mathrm{i}}, v_{\mathrm{i}}\right)\left(u_{\mathrm{i}}{ }^{\prime}, v_{\mathrm{i}}{ }^{\prime}\right)$, respectively.

$$
\text { dis }=\sqrt{\left(u_{\mathrm{i}}^{\prime}-u_{\mathrm{i}}\right)^{2}+\left(v_{\mathrm{i}}^{\prime}-v_{\mathrm{i}}\right)^{2}}
$$




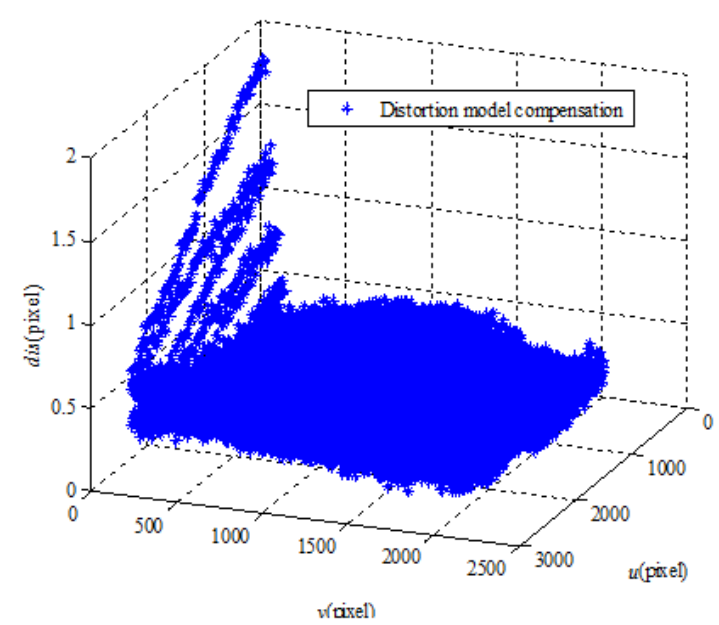

Fig. 5 Distances between ideal imaging points and those obtained after model compensation

In Fig. 5 plane $u v$ is the ideal image plane. The vertical axis dis represents the distance between ideal image pixel coordinates and those after compensation based on distortion model. It can be seen from Fig. 5 that there are a few lines with dis greater than 1 pixel where $v$ is near to zero. After several tests results are basically the same. It can be concluded that these errors are not caused by the noises of the camera, but by distortions of the camera and the imperfection of the model based on Eq. (1) in the boundary area.

\subsection{Distortion Database Compensation Evaluation}

The error caused by parameters $d_{x}, k_{1}, k_{2}, c_{x}, c_{y}$ is considered as the distortion error. The error caused by the focal length $f$ is the magnification error. A method for separating the distortion error and magnification error is proposed in this paper.

In Fig. $6 P$ is a point with coordinates $\left(x_{\mathrm{w},}, y_{\mathrm{w}}, z_{\mathrm{w}}\right)$ in the world system and coordinates $\left(x_{\mathrm{c}}, y_{\mathrm{c}}, z_{\mathrm{c}}\right)$ in the camera system. $p_{\mathrm{i}}$ is its image. $p_{\mathrm{id}}$ with coordinates $\left(x_{\mathrm{wd}}, y_{\mathrm{wd}}, z_{\mathrm{wd}}\right)$ in the world system is the pedal of the point $P$ to the line $o_{c} p_{\text {i }}$. The distance $d d$ between point $P$ and point $p_{\text {id }}$ can be served as the index for evaluating the distortion error and

$$
d d=\sqrt{\left(x_{\mathrm{wd}}-x_{\mathrm{w}}\right)^{2}+\left(y_{\mathrm{wd}}-y_{\mathrm{w}}\right)^{2}+\left(z_{\mathrm{wd}}-z_{\mathrm{w}}\right)^{2}}
$$

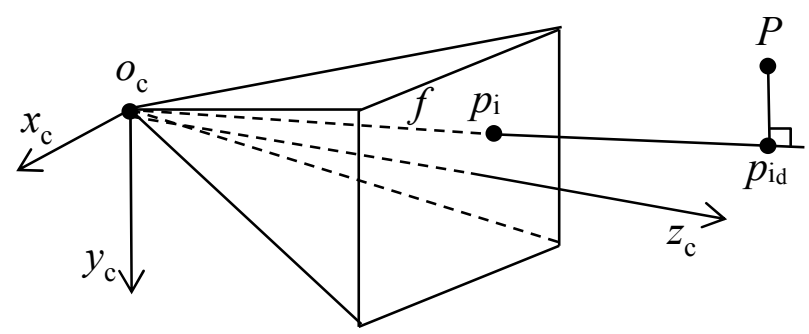

Fig. 6 Distortion error evaluation

To compare the effects of the proposed method based on distortion database and method based on distortion model and to analyze the influences of various factors on the compensation accuracy six sets of tests shown in Table 2 were carried out. The parameters listed in the third column of Table 2 are those used in the iteration process of distortion model compensation. Those given in the fourth column are parameters used in the process of establishing database. 
Table 2. Parameters used in six tests

\begin{tabular}{|c|c|c|c|}
\hline Test & Parameters calibrated & $\begin{array}{c}\text { Parameters used in } \\
\text { iteration }\end{array}$ & $\begin{array}{c}\text { Parameters used in building } \\
\text { database }\end{array}$ \\
\hline$a 0$ & none & none & none \\
\hline$a 1$ & $\boldsymbol{R}, \boldsymbol{T}, f, c_{x}, c_{y}, k_{1}, k_{2}, \theta, \varphi$, & $c_{x}, c_{y}, k_{1}, k_{2}, \theta, \varphi, d_{x}$ & none \\
\hline$a 2$ & $d_{x}$ & none & $\boldsymbol{R}, \boldsymbol{T}, f, c_{x}, c_{y}, d_{x}$ \\
\hline$a 3$ & $\boldsymbol{R}, \boldsymbol{T}$ & none & $\boldsymbol{R}, \boldsymbol{T}, f, c_{x}, c_{y}, d_{x}$ \\
\hline$a 4$ & $\boldsymbol{R}, \boldsymbol{T}, f$ & none & $\boldsymbol{R}, \boldsymbol{T}, f, c_{x}, c_{y}, d_{x}$ \\
\hline$a 5$ & $\boldsymbol{R}, \boldsymbol{T}, f, c_{x}, c_{y}, c_{1}, c_{2}, \theta, \varphi, d_{x}$ & $c_{x}, c_{y}, k_{1}, k_{2}, \theta, \varphi, d_{x}$ & $\boldsymbol{R}, \boldsymbol{T}, f, c_{x}, c_{y}, k_{1}, k_{2}, \theta, \varphi, d_{x}$ \\
\hline
\end{tabular}

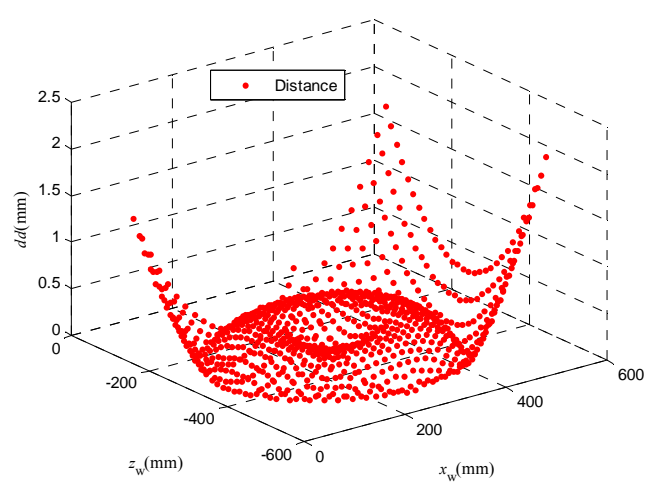

$\mathrm{a} 0$

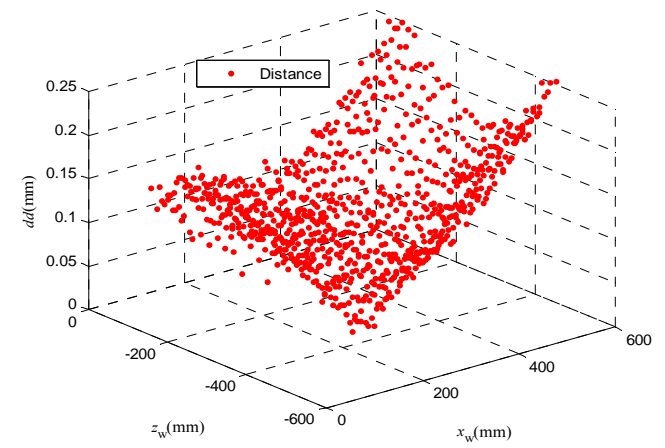

a2

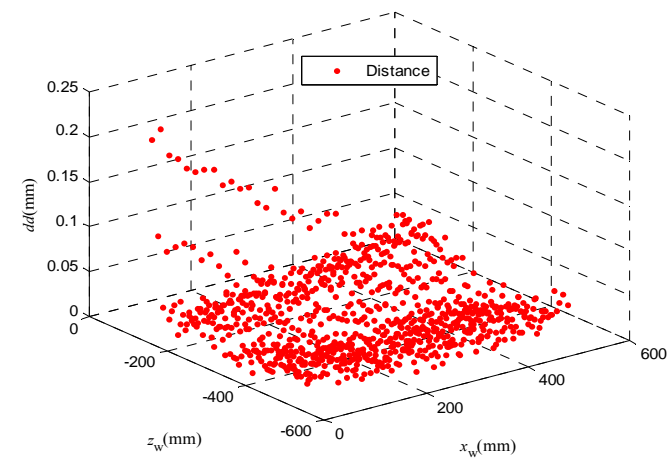

a4

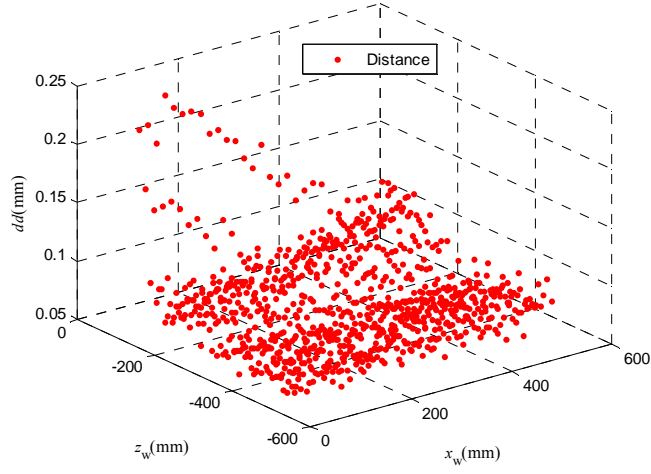

a1

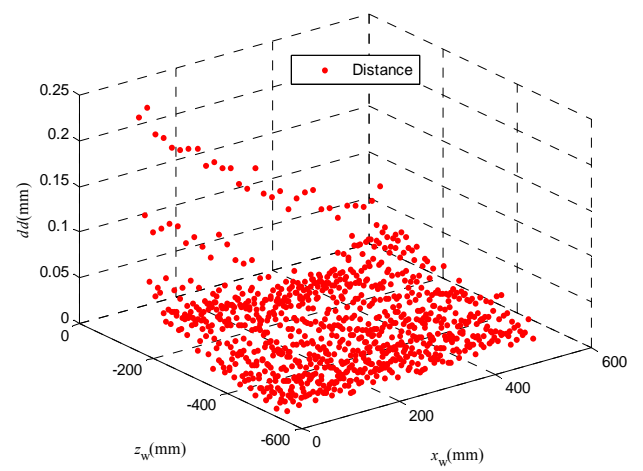

a3

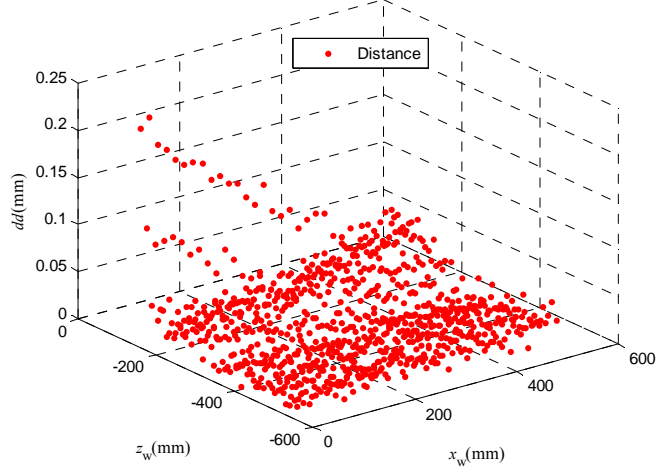

a5

Fig. 7 The distances dd obtained in six tests

In all tests the initial value of focal length $f$ is the labeled value of the camera, the initial coordinates $\left(c_{x}, c_{y}\right)$ of the principal point are center pixel coordinates of the image plane, and the initial value of $d_{x}$ is the value given in the manual of camera. Test $a 0$ is a test without any camera calibration and distortion compensation. In test $a 1$ the calibration and compensation are based on distortion model. All the camera parameters $\boldsymbol{R}, \boldsymbol{T}, f, c_{x}, c_{y}, k_{1}, k_{2}, \theta, \varphi, d_{x}$ are calibrated and compensation is introduced in accordance with Eq. (1). No compensation based on distortion 
database is performed. To analyze factors affecting the accuracy of compensation based on the distortion database, four tests $a 2$ to $a 5$ are performed. In test $a 2$ only $\boldsymbol{R}$ and $\boldsymbol{T}$ are calibrated. The initial values of $f, c_{x}, c_{y}, d_{x}$ and $k_{1}=k_{2}=\theta=\varphi=0$ are adopted for calculating $d d$. In test $a 3$ the focal length $f$ is calibrated additionally in comparison with $a 2$. In test $a 4$, compared with $a 3$, coordinates $\left(c_{x}, c_{y}\right)$ of the principal point and $d_{x}$ are calibrated additionally. In test $a 5$ four more parameters $k_{1}, k_{2}, \theta, \varphi$ are calibrated in comparison with $a 4$, which means all the parameters in Eq. (1) are calibrated. Distortion databases for $a 2, a 3, a 4$ and $a 5$ are established based on differences between the coordinates of ideal image points and those after compensation based on distortion model.

It can be seen from Fig. 7 due to the imperfection of the optical system distances $d d$ at the edges of the image plane are significant. Distortion database compensation and distortion model compensation are only able to compensate the distortions to certain extent. The distortions at the edges are quite large and the distortion curve in this area is steep. The slight change in the position of the image point would cause significant change of the distortion value. It is difficult to accurately calibrate distortions at the edges of image plane. Besides the distortion curve in this area is nonlinear. Linear interpolation causes some additional error in distortion database compensation

It is recommended not to use the edge zone of the image plane in practical measurement. Points at the edges of the image plane with large errors are removed, and the average distances $d d$ of remaining 2600 points are shown in Fig. 8. The vertical axis in Fig. $8 d d v$ denotes the average distances of these 2600 points. Red dot indicates the average distance $d d v$ of test $a 0$ without any compensation. Blue dot indicates that of the test $a 1$ after distortion model compensation. Black dots indicate those of tests after four types of distortion database compensation.

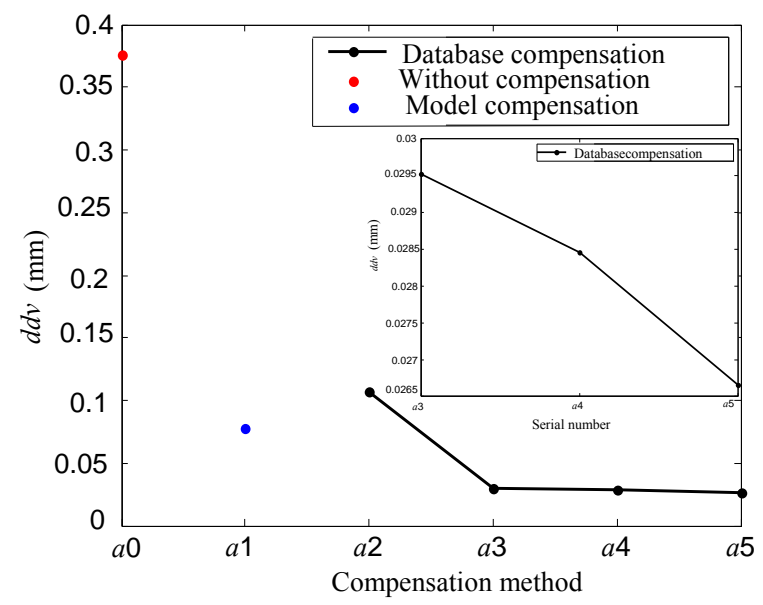

Fig. 8 The average distance difference of six methods

\section{Conclusion}

(1) A new CMM based method of high-precision camera calibration and distortion compensation is proposed in this paper. A high accurate three-dimensional reference with large number of feature points is provided by the CMM. Such a $3 \mathrm{D}$ reference is flexible in terms of spacing and distribution of feature points and is able to cover the entire image plane of the camera.

(2) A new method for distortion compensation based on distortion database is presented. Experiments show that it is superior to common used method based on distortion model. The imperfection of model has little effect on the accuracy of compensation based on distortion database. It can be used directly after $\boldsymbol{R}, \boldsymbol{T}$ and $f$ have been calibrated. However it gives even smaller final errors after compensation when the distortion model compensation is carried out first.

(3) An index for evaluating the error caused by distortion of camera is put forward. It equals the distance between the measured point $P$ and $p_{\text {id }}$ where $p_{\text {id }}$ is the pedal of point $P$ on line connecting the optical center $o_{\mathrm{c}}$ and image point $p_{\mathrm{i}}$ of point $P$. 


\section{Acknowledgments}

This work is performed under the grant No. 13JCZDJC34500 supported by the Natural Science Foundation of Tianjin. The authors would like to express their sincere thanks to the Natural Science Foundation of Tianjin.

\section{References}

[1]. Brown, D. C. Close-range camera calibration. Photogrammetric Engineering, 37 (8) (1971), 855-866,.

[2]. Faig. W. Calibration of close- range photogrammetry system: mathematical formulation. Photogrammetric Engineering and Remote Sensing, 41 (12) (1975), 1479-1486.

[3]. Weng, J., Cohen, P., Hemion, M. Camera calibration with distortion models and accuracy evaluation. IEEE Transactions on Pattern Analysis \& Machine Intelligence, 14(10) (1992), 965-980.

[4]. Wang, J. H., Shi, F. H., Zhang, J., Liu, Y. C. A new calibration model of camera lens distortion, Pattern Recognition, 41(2) (2008), 607-615.

[5]. Weng, J. Y., Cohen, P., Herniou, M, Calibration of stereo cameras using a non-linear distortion model. Pattern Recognition, (1990), 246-253.

[6]. Lenz, R. K., Tsai, R. Y. Techniques for calibration of the scale factor and image center for high accuracy 3D machine vision metrology. International Conference on Robotics and Automation, Raleigh, North Carolina, March .IEEE, (1987), 68-75.

[7]. Tsai, R. Y. A versatile camera calibration technique for high accuracy 3D machine vision metrology using off-the-shelf TV cameras and lenses. Journal of Robotics and Automation, 3(4) (1987). IEEE. 323-344.

[8]. Li, D., Tian, J. D. An accurate calibration method for a camera with telecentric lenses. Optics and Lasers in Engineering, 51(5), (2013), 538-541.

[9]. Sobel, I. On calibrating computer controlled cameras for perceiving 3-D scenes. Artificial Intelligence, 5(2) (1974), 185-198.

[10]. Gennery, G. B. Stereo-camera calibration. Image Understanding workshop, (1979), 101-108.

[11]. Hakan B., Mohanmed S. K. A three-step camera calibration method. Transactions on instrumentation and measurement, 46(5) (1997), IEEE, 1165-1172.

[12]. Hartley, R. I. An algorithm for self-calibration from several views. Conf. Computer Vision and pattern recognition, IEEE, (1994), 908-912.

[13]. Hartley, R. I. Self-calibration from multiple views with a rotating camera. Third European Conference on Computer Vision Stockholm, (1994), 471-478.

[14]. Zhang, Z. Y. A flexible new technique for camera calibration. Transactions on Pattern Analysis and Machine Intelligence, 22(11) (2000), IEEE, 1330-1334.

[15]. Zhang, G. J., He, J .J. Yang, X. M. Calibrating camera radial distortion with cross-ratio invariability. Optics \& Laser Technology, 35(6) (2003). 457-461.

[16]. Yang, Z. J., Chen, F., Zhao, J., Zhao, H. W., A novel camera calibration method based on genetic algorithm. Industrial Electronics and Applications IEEE(2008), 2222-2227.

[17]. Ahmed, M., Farag, A. Nonmetric calibration of camera lens distortion: differential methods and robust estimation. Image Process. 14 (8) (2005), IEEE, 1215-1230. 